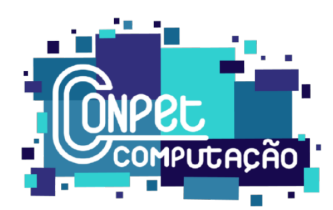

\title{
Introdução de indígenas e quilombolas aos ambientes digitais de ensino da universidade
}

\author{
Gerônimo G. Z. Lemos ${ }^{1}$, Bruno C. Alves ${ }^{1}$, Heitor F. M. dos Santos ${ }^{1}$, \\ Gerson L. de Menezes ${ }^{1}$, Leomar S. da Rosa Júnior ${ }^{1}$ \\ ${ }^{1}$ Centro de Desenvolvimento Tecnológico \\ Universidade Federal de Pelotas (UFPel) - Pelotas - RS - Brasil \\ \{ggzlemos, bcalves, hfmdsantos, gldmenezes, leomarjr\}@inf.ufpel.edu.br
}

\begin{abstract}
This work investigates the obstacles faced by Indigenous and Quilombo students at the Federal University of Pelotas (UFPel) when referring to academic tasks during the COVID-19 pandemic. With the need to switch over to remote activities, several students from these communities suffered problems because they did not have access to the teaching platforms. Therefore, a dialogue channel was created between Indigenous and Quilombo students, Affirmative Activities and Diversity Core, and PET Computação from UFPel, aiming to investigate the challenges the students go through and help them to get comfortable with the UFPel's virtual tools. Lastly, it is proposed the creation of an assistance system aiming at resources for those students who do not have access to technology.
\end{abstract}

Resumo. Este trabalho busca analisar os obstáculos enfrentados por alunos indígenas e quilombolas da Universidade Federal de Pelotas (UFPel) referente à realização de tarefas acadêmicas durante a pandemia de COVID-19. Com a adesão às atividades remotas, diversos alunos dessas comunidades enfrentaram problemas por não terem acesso às plataformas de estudo. Por isso, criou-se um canal de diálogo entre os alunos indígenas/quilombolas, membros do Núcleo de Ações Afirmativas e Diversidade e grupo PET Computação da UFPel, a fim de investigar os desafios encontrados pelos alunos, além de auxiliar no uso de ferramentas digitais da UFPel. Por fim, propõem-se a criação de auxílios para a viabilização de recursos aos alunos que não possuem acesso à tecnologias.

\section{Introdução}

Atualmente as tecnologias digitais têm grande importância no que diz respeito ao acesso à informação, relações sociais e educação, particularmente durante a pandemia de COVID19, em que, segundo as autoridades em saúde, deve-se praticar distanciamento social. Assim, aspectos do dia-a-dia como trabalho, estudos, relações familiares e sociais são realizados com o auxílio da tecnologia. Isso vai ao encontro do conceito de [Jaeger et al. 2012], que define inclusão digital como uma política desenvolvida para promover a integração virtual com populações não atendidas e carentes. Em sentido oposto, define-se o termo exclusão digital, que faz referência à situação dos indivíduos que não possuem acesso às tecnologias da informação e estão à margem do desenvolvimento tecnológico. Ainda, também se faz presente a exclusão digital, sempre que há acesso a essas tecnologias mas os indivíduos não possuem o conhecimento necessário para a utilização das novas tecnologias. 
Devido à pandemia de COVID-19, as instituições de ensino superior no Brasil suspenderam suas atividades presencias, de forma a seguir e estimular o distanciamento social [Organization et al. 2020]. Dessa forma, as atividades acadêmicas nessas instituições foram adaptadas a esse momento de isolamento, sendo realizadas de forma remota. Assim, com a adoção dessa nova modalidade de ensino, evidenciou-se o grande número de estudantes do ensino superior que são excluídos da utilização dos avanços tecnológicos. Segundo [Nascimento et al. 2020], no ano de 2018, o número de alunos de ensino superior que não possuíam acesso domiciliar à Internet de banda larga ou $3 \mathrm{G} / 4 \mathrm{G}$, somados os cursos de graduação e pós-graduação strictu-sensu, chega perto dos 200 mil.

A Universidade Federal de Pelotas (UFPel), uma das primeiras instituições de ensino superior a suspender suas atividades presencias, iniciou suas atividades remotas após consulta à sua comunidade estudantil. Para atender essa finalidade, a universidade disponibilizou aos alunos e professores as plataformas e-aula, para realização das atividades acadêmicas, e web-conf, para a realização dos encontros síncronos entre alunos e professores. Porém, alguns alunos ainda possuem dificuldades técnicas e práticas para acessar esses ambientes virtuais e servir-se deles. Notoriamente, destacam-se dentre esse grupo, os alunos indígenas e quilombolas. Muitos desses alunos não possuem acesso às ferramentas digitais necessárias para a realização das atividades. Entretanto, essa não é a única dificuldade encontrada por esses alunos. Muitos dos que possuem acesso às ferramentas necessárias não sabem como se servir delas para realização das atividades.

Segundo o Núcleo de Ações Afirmativas da UFPel (NUUAD), a UFPel conta com 71 alunos das comunidades indígenas e quilombolas, distribuídos em 24 cursos. Além disso, a UFPel garante 50\% das matrículas de cada curso para alunos indígenas, negros, pardos e de baixa renda, nos termos do que determina a Lei federal $n^{\circ} 12.711 / 2012$, também conhecida como Lei das Cotas. Percebe-se que nos últimos anos, há maior preocupação em relação à representatividade dessa população dentro das universidades. Diante disso, percebe-se a grande importância de ações institucionais para maior integração desses estudantes dentro das universidades. O presente trabalho busca investigar quais são os principais obstáculos enfrentados pelos alunos indígenas e quilombolas da UFPel diante do novo modelo de ensino digital adotado por essa universidade para o período da pandemia de COVID-19. Entender as dificuldades desses alunos é de grande importância para a elaboração de ações integradoras que auxiliem esses estudantes a se desenvolver dentro da universidade.

Este artigo está estruturado da seguinte maneira: a Seção 2 apresenta alguns antecedentes importantes para este trabalho; a Seção 3 apresenta o desenvolvimento do trabalho; a Seção 4 apresenta os resultados e as discussões; e, por fim, a Seção 5 apresenta as conclusões deste trabalho.

\section{Estado da Arte}

Devido à pandemia de COVID-19, os estudantes do ensino superior no Brasil enfrentam problemas para realizar suas atividades acadêmicas, em função das dificuldades decorrentes da adesão do ensino remoto pelas instituições de ensino, adotadas para que se estimule o distanciamento social. Destacam-se para finalidade desse trabalho os estudantes indígenas e quilombolas, que historicamente, estão à margem do desenvolvimento tecnológico no Brasil. Tendo em vista as dificuldades enfrentadas por esses alunos, expostas 
na seção anterior, serão revisados os dados presentes na literatura para auxiliar o entendimento dos problemas enfrentados por esses grupos, a fim de se buscar uma solução específica para os alunos da UFPel.

Segundo [Nascimento et al. 2020], no ano de 2018, aproximadamente 200 mil alunos de ensino superior que não possuíam acesso domiciliar à Internet de banda larga ou 3G/4G, somados os cursos de graduação e pós-graduação strictu-sensu. Dentre os alunos sem acesso à Internet, a maioria é de negros e indígenas. Esses dados expõem como, mesmo em uma sociedade altamente tecnológica, muitas pessoas ainda não têm acesso a essas tecnologias e, portanto, ficam à margem do desenvolvimento tecnológico.

De acordo com [Grossi et al. 2013], a exclusão digital tem como produto a desigualdade social, que, consequentemente, estimula a exclusão digital. Garantir que todos os alunos da UFPel tenham acesso às suas ferramentas digitais durante o período de isolamento social é fazer cumprir o papel dessa universidade como instituição de ensino diversa e democrática.

O trabalho de [Babrosa and da Cunha 2020] analisa de forma ampla a exclusão digital dos alunos de baixa classe social no Brasil e as consequências que isso tem para a manutenção da desigualdade social no Brasil. Para isso, esses autores analisam dados do Instituto Brasileiro de Geografia e Estatística (IBGE) referentes ao acesso à Internet das diferentes classes sociais do país. Segundo os autores, durante o isolamento social, as diferenças entre estudantes de diferentes classes sociais aumenta.

Em seu artigo, [Selleri et al. 2013] faz um levantamento do uso de ferramentas digitais por comunidades indígenas. Nesse trabalho, o autor visita 5 comunidades no estado do Mato Grosso do Sul e investiga a relação que os indígenas dessas comunidades têm com a tecnologia, bem como quais são os recursos que eles possuem.

Já em seu artigo, [Aguilar 2012] analisa a identidade e diversidade cultural de duas tribos indígenas no ambiente digital. Para isso, analisa duas tribos do Nordeste do país e como são as suas relações com as tecnologias da informação (TICs). Além disso, a autora analisa como o uso das TICs influencia na construção e compartilhamento da cultura das tribos analisadas. Por fim, investiga quais são as principais atividades realizadas pelas tribos com o auxílio das TICs. Dentre essas atividades, predominam atividades escolares, de pesquisa sobre culturas de diferentes tribos, além de entretenimento.

A partir da análise dos artigos citados acima, o presente trabalho tem como objetivo investigar as principais dificuldades enfrentadas pelos alunos indígenas e quilombolas da UFPel, com o objetivo de disponibilizar um melhor suporte a esses alunos na utilização das ferramentas digitais ofertadas pela UFPel durante o período de isolamento social. Notoriamente, esses grupos de alunos são excluídos dos benefícios dos avanços tecnológicos que o restante da sociedade tem acesso, contribuindo para o aumento da desigualdade social. Para isso, entender os obstáculos enfrentados por esses alunos é de grande importância para que ações sejam elaboradas para melhor atender essa comunidade. Atender as demandas desses grupos torna-se de grande importância para a UFPel, para que a universidade possa cumprir o seu papel de instituição de ensino de qualidade. 


\section{Trabalho Desenvolvido}

Para o desenvolvimento do presente trabalho, foi realizado um encontro entre os alunos das comunidades indígenas e quilombolas da Universidade Federal de Pelotas (UFPel), membros do Núcleo de Ações Afirmativas e Diversidade da universidade (NUAAD) e alunos membros do Programa de Ensino Tutorial dos cursos de Ciência e Engenharia da Computação da mesma universidade (PET Computação). Nesse encontro, discutiu-se o acesso dos estudantes às plataformas digitais oferecidas pela universidade, bem como as principais dificuldades de acesso enfrentadas por eles. Segundo o NUAAD, atualmente a UFPel conta com 71 alunos indígenas e quilombolas, distribuídos em 24 cursos.

Devido à necessidade de isolamento social em função da pandemia de COVID-19, optou-se pela realização de um encontro remoto, utilizando a plataforma Google Meet. Justamente devido às dificuldades enfrentadas pelos alunos indígenas e quilombolas, o encontro foi realizado com número reduzido de alunos dessas comunidades. Dados adicionais foram disponibilizados pelo NUAAD. Além disso, foram estruturados encontros futuros para que os alunos ausentes pudessem participar.

No encontro, os alunos do grupo PET abordaram as principais dificuldades enfrentadas pelos alunos indígenas e quilombolas, com o objetivo de auxiliar esse grupo de alunos a realizar suas atividades acadêmicas durante o período de distanciamento social. Também foi criado um canal de comunicação entre o grupo PET e os alunos participantes por meio de plataforma de troca de mensagens, com o objetivo de facilitar o contato entre as partes.

Além disso, foram levantados dados quantitativos no que diz respeito ao acesso dos alunos indígenas e quilombolas às ferramentas digitais. A Figura 1 apresenta dados relativos aos alunos com disponibilidade de um computador ou celular para a realização do encontro. Dos 71 alunos das comunidades indígenas e quilombolas da UFPel, 13 discentes possuem computador ou celular disponível para a realização do encontro com os alunos do grupo PET. Ou seja, cerca de $80 \%$ dos alunos não tiveram condições de participar do encontro.

Além da falta de acesso às ferramentas que permitem que os alunos indígenas e quilombolas possam participar integralmente das atividades acadêmicas, muitos desses alunos, quando têm acesso a essas ferramentas, não sabem como utilizar-se delas. No encontro realizado, foram discutidas as principais dificuldades encontradas pelos alunos na utilização das plataformas e-aula e web-conf, ambas disponibilizadas pela universidade para o decorrer dos semestres em que o distanciamento social seja necessário. Os tópicos abordados no encontro foram fortemente baseados nas dúvidas dos estudantes, compreendendo questões relacionadas à utilização do ambiente digital de aprendizado da UFPel, como: envio de arquivos, submissão de atividades aos professores, realização de testes, comunicação com colegas e docentes através de chat (fórum de discussão), áudio e vídeo. Com isso, nota-se que as dificuldades encontradas por esses alunos não se restringem apenas à falta de acesso às ferramentas digitais básicas, como o computador ou acesso à Internet de qualidade, mas também abrangem a utilização dos recursos disponibilizados pela universidade.

Com a realização do encontro entre os alunos e o levantamento dos dados do NUAAD, foi possível ter uma visão mais clara das principais dificuldades encontradas 


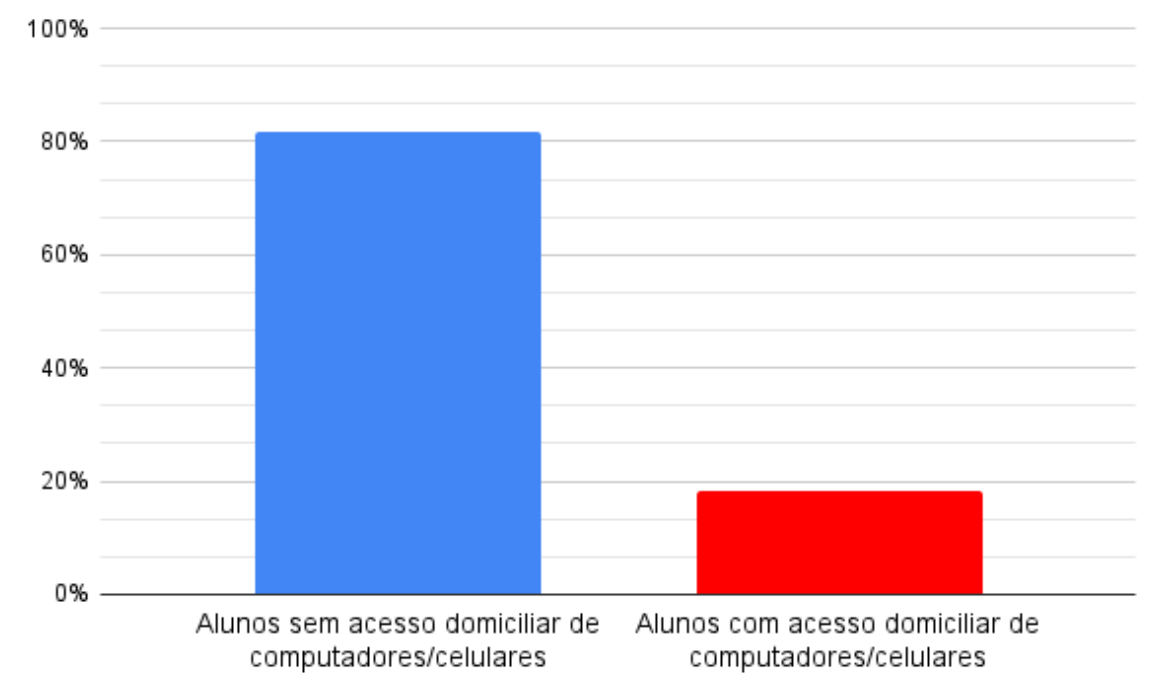

Figura 1. Porcentagem de alunos indígenas e quilombolas da UFPel com/sem acesso à computadores ou celulares.

por esses alunos. A dificuldade desses alunos não se resume apenas à falta de recursos computacionais para a realização das atividades, mas também falta conhecimento sobre a utilização dos recursos oferecidos.

Portanto, propõem-se à gestão da universidade um programa de auxílio para a viabilização de recursos computacionais aos alunos. Tendo em vista que grande parte dos alunos das comunidades indígenas e quilombolas ainda não possuem os recursos necessários para a realizar suas atividades durante os semestres realizados remotamente, esse tipo de ação torna-se fundamental. Além disso, encontros presenciais tornam-se necessários para o melhor diagnóstico da situação desses alunos. Para isso, propõemse a realização de encontros domiciliares que respeitem as normas de segurança sanitária. Então, são necessários recursos da universidade, como meios de transporte, equipamentos de segurança e álcool em gel. Conseguindo tais recursos e realizando esses encontros, será possível ter uma visão mais precisa da atual situação dos alunos, sendo possível, assim, oferecer um melhor auxílio.

\section{Resultados e Discussões}

Ao realizar o encontro com os estudantes, foram abordadas as principais dificuldades encontradas por estes ao executar as atividades remotas. Muito dessas dificuldades são consequências do pouco contato que esses alunos têm com essas tecnologias. Além disso, muitos alunos não participaram da reunião por não possuir a disponibilidade dos recursos computacionais ou de Internet no horário da realização do encontro.

Segundo os dados fornecidos pelo NUAAD, a grande maioria dos alunos não possuem acesso às ferramentas necessárias para participar plenamente das atividades remotas. Esse fator contribui para a falta de familiaridades dos alunos com as ferramentas, o que, consequentemente, afeta a sua experiência na instituição.

Com a realização deste primeiro encontro, ilustrado pela Figura 2 , destaca-se a criação de um canal de comunicação direto entre NUAAD, PET Computação e os alu- 


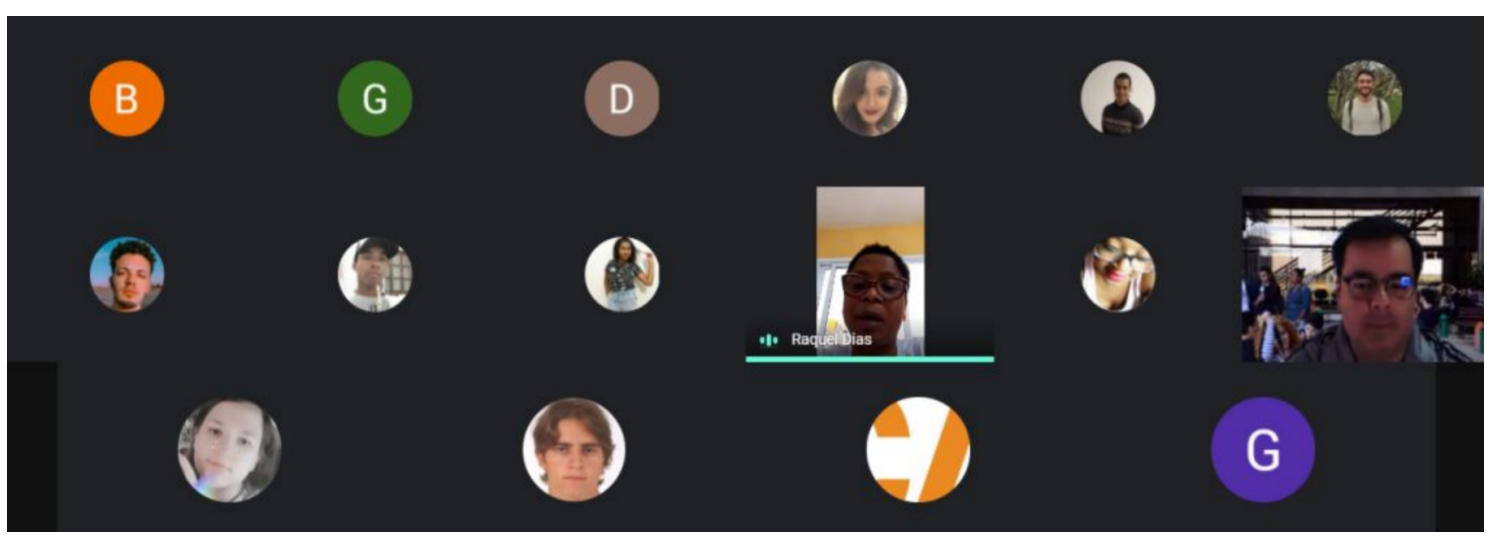

Figura 2. Registro do encontro entre alunos indígenas e quilombolas, PET Computação e NUAAD.

nos indígenas e quilombolas. Dessa forma, os alunos têm acesso direto aos membros do grupo PET, o que possibilita a eles um auxílio mais dinâmico. Propõem-se, então, a realização de encontros presencias com esses alunos, para que sejam identificadas as principais dificuldades encontradas por eles. Sendo assim, busca-se recursos da universidade, como transporte e equipamentos de segurança, para que tais encontros sejam realizados seguindo as normas de proteção sanitárias. Ações como essa são de grande importância para a inclusão desses alunos nos ambientes digitais da universidade.

Além disso, o pleno entendimento das dificuldades desses alunos é de extrema importância para a elaboração de ações institucionais por parte da universidade para dar suporte a todos os alunos que não possuem acesso às tecnologias. Em consequência do cenário atual, sugere-se aqui a criação de um programa de auxílio específico para aquisição de recursos computacionais direcionado aos alunos que não possuem acesso. Juntamente a isso, o fomento a projetos de ensino que proporcionem o aprendizado do manuseio desses recursos também se mostra importante.

Por fim, destaca-se a necessidade de um olhar mais atento às dificuldades enfrentadas pela comunidade discente da universidade durante a pandemia de COVID-19. A universidade, como instituição prestadora de serviço de ensino de qualidade, deve estar atenta às necessidades dos alunos durante esse período. Portanto, torna-se fundamental a realização de ações institucionais e projetos de ensino que incentivem a integração desses alunos na instituição.

\section{Conclusões}

O presente trabalho analisou as principais dificuldades na realização as atividades acadêmicas dos alunos indígenas e quilombolas da Universidade Federal de Pelotas (UFPel) no contexto da pandemia de COVID-19. Devido ao isolamento social, necessário durante esse período, a universidade adotou a modalidade de ensino remoto. Esses alunos, que notoriamente têm acesso restrito às tenologias da informação, apresentam grande dificuldade para realizar suas atividades acadêmicas durante esse período.

Para o desenvolvimento desse trabalho, foi realizado um encontro entre os alunos indígenas e quilombolas da UFPel, membros do grupo PET Computação e membros do Núcleo de Ações Afirmativas e Diversidade (NUAAD) da universidade. Nesse encon- 
tro, foram discutidas as principais dificuldades encontradas por esses alunos. Além disso, foram esclarecidas dúvidas sobre o uso dos sistemas e-aula e webconf, ambos disponibilizados pela UFPel para a realização das atividades remotas. Destaca-se aqui, a criação de um canal de comunicação direto entre os participantes desse encontro. Com isso, os alunos indígenas e quilombolas têm maior auxílio para a realização de suas atividades. Finalmente, foram recolhidos dados do NUUAD, que dizem respeito ao número de alunos indígenas e quilombolas da UFPel.

Com o retorno das atividades acadêmicas ao modo presencial, previsto para o ano de 2022, existe a pretensão de continuidade no projeto UFPel Digital. Com isso, os encontros serão feitos presencialmente nos laboratórios dos cursos de engenharia e ciência da computação, os quais oferecem todos os recursos necessários para o ensino e aprendizado de ferramentas digitais. Além disso, serão discutidas ferramentas digitais que auxiliam no desenvolvimento acadêmico.

Finalmente, destaca-se a importância de ações institucionais que auxiliem esses alunos à realizarem suas atividades. Notoriamente, esses alunos possuem menor acesso às tecnologias da informação, o que contribui para que eles tenham maior dificuldade. Portanto, torna-se imprescindível que a universidade elabore ações para melhor auxiliar esses alunos.

\section{Referências}

Aguilar, A. (2012). Identidade/diversidade cultural no ciberespaco: praticas informacionais e de inclus? o digital nas comunidade indigenas no brasil. Informação \& Sociedade, 22(1).

Babrosa, O. L. and da Cunha, P. G. M. (2020). Pandemia e a precarização do direito ao acesso à educação. Revista Pet Economia UFES, 1(1):33-36.

Grossi, M. G. R., da Costa, J. W., and dos Santos, A. J. (2013). A exclusão digital: o reflexo da desigualdade social no brasil. Nuances: estudos sobre Educação, 24(2):6885 .

Jaeger, P. T., Bertot, J. C., Thompson, K. M., Katz, S. M., and DeCoster, E. J. (2012). The intersection of public policy and public access: Digital divides, digital literacy, digital inclusion, and public libraries. Public library quarterly, 31(1):1-20.

Nascimento, P. M., Ramos, D. L., Melo, A. A. S. d., and Castioni, R. (2020). Acesso domiciliar à internet e ensino remoto durante a pandemia.

Organization, W. H. et al. (2020). Considerations in adjusting public health and social measures in the context of covid-19: interim guidance, 16 april 2020. Technical report, World Health Organization.

Selleri, F., Januário, E., Silva, G. S., de Oliveira, C. Q., do Nascimento, E. R., and Azevedo, I. M. (2013). Inclusão digital em escolas e comunidades indígenas. In Anais do Workshop de Informática na Escola, volume 1, page 437. 